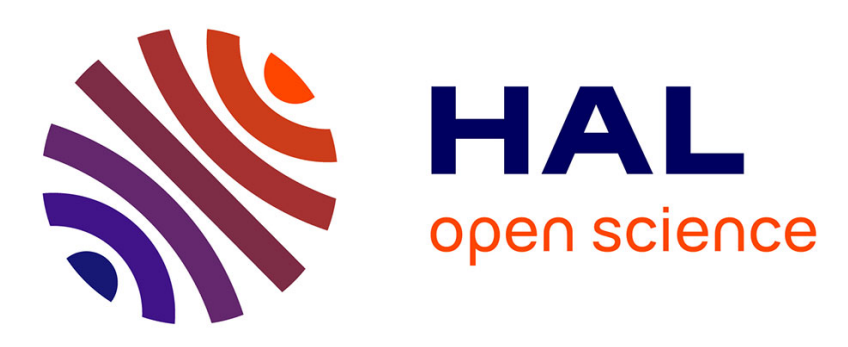

\title{
Reliable Eyelid Localization for Iris Recognition
}

Mathieu Adam, Florence Rossant, Frédéric Amiel, Beata Mikovicova, Thomas Ea

\section{To cite this version:}

Mathieu Adam, Florence Rossant, Frédéric Amiel, Beata Mikovicova, Thomas Ea. Reliable Eyelid Localization for Iris Recognition. 10th International Conference on Advanced Concepts for Intelligent Vision Systems (ACIVS 2008), Oct 2008, Juan les Pins, France. pp.1062-1070, 10.1007/978-3-54088458-3_96. hal-02882946

\section{HAL Id: hal-02882946 https://hal.science/hal-02882946}

Submitted on 28 Aug 2020

HAL is a multi-disciplinary open access archive for the deposit and dissemination of scientific research documents, whether they are published or not. The documents may come from teaching and research institutions in France or abroad, or from public or private research centers.
L'archive ouverte pluridisciplinaire HAL, est destinée au dépôt et à la diffusion de documents scientifiques de niveau recherche, publiés ou non, émanant des établissements d'enseignement et de recherche français ou étrangers, des laboratoires publics ou privés. 


\title{
Reliable Eyelid Localization for Iris Recognition
}

\author{
Mathieu Adam, Florence Rossant, Frederic Amiel, \\ Beata Mikovicova, and Thomas Ea \\ ISEP, 21 Rue d'Assas, 75006 Paris, France \\ \{mathieu.adam, florence.rossant, frederic.amiel, \\ beata.mikovicova, thomas.ea\}@isep.fr \\ http://www.isep.fr
}

\begin{abstract}
This article presents a new eyelid localization algorithm based on a parabolic curve fitting. To deal with eyelashes, low contrast or false detection due to iris texture, we propose a two steps algorithm. First, possible edge candidates are selected by applying an edge detection on a restricted area inside the iris. Then, a gradient maximisation is applied along every parabola, on a larger area, to refine the parameters and select the best one. Experiments have been conducted on the CASIAIrisV3-Interval database that have been manually segmented. A new performance measure is proposed, carried out by comparing the segmented images obtained by the proposed method with the manual segmentation.
\end{abstract}

Key words: biometric identification, iris analysis, eyelid segmentation, edge selection, curve fitting

\section{Introduction}

Biometric is progressively replacing traditional identification methods such as electronic key or password. Among different biometric technologies, iris recognition is considered as the most reliable application [1].

Major difficulties come from the poor quality of the acquired images. They are often blurred, defocused or occulted by eyelids. Several authors propose to analyse the image quality in order to select the best image from an acquisition sequence [2],[3]. However, an efficient iris localization is still required, since the whole recognition system depends on the accuracy of this segmentation step.

In particular, the detection of eyelid occlusions is crucial to achieve good recognition rates. But it is a very difficult issue, since eyelashes often hide the eyelid boundaries. Also, the iris and eyelid regions may be difficult to separate, because of a very low contrast or a highly textured iris. Some eyelid localization methods have already been studied in [2],[4],[5],[6],[7], but the criteria used to determine the segmentation accuracy are generally not much detailed.

This paper presents a novel eyelid localization method and focuses on measuring with objectivity the segmentation quality. For that, we define two kinds of errors, sub-segmentation and over-segmentation, and we evaluate the segmentation quality by comparing manual and automatic eyelid segmentation on the CASIA-IrisV3 database, containing 2655 images. 
The paper is organized as follows: section 2 describes briefly the global iris recognition system. Section 3 focuses on our new eyelid localization method. Finally, experimental results are presented in section 4 .

\section{Global Recognition System}

A complete iris recognition system has been already presented in [8],[9]. It is divided in three parts (Fig. 1). The preprocessing part includes the iris segmentation and unwrapping (a). Then, a third level wavelet packet decomposition is applied to the unwrapped iris to generate the signature (b). Finally, the recognition process is based on a distance calculation, in order to compare the extracted signature with the ones stored in a reference database (c).

The system is effective and robust to illumination, blurring, optical axis deviation and local defects [9]. But it can be improved by taking into account possible eyelid occlusions.

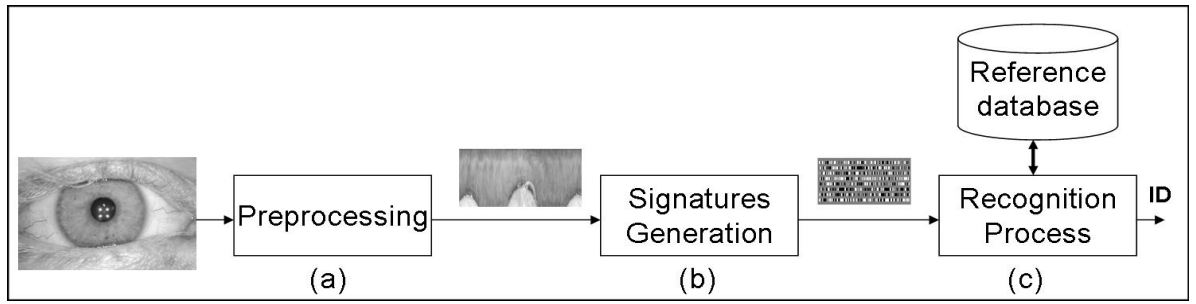

Fig. 1. Global recognition system

\section{Eyelid Localization}

\subsection{Iris Localization}

The iris localization algorithm is performed with a gradient based search method [9]. The pupil is modeled by a circle and the outer iris boundary by an ellipse. Both curves are found by maximizing the mean gradient in the orthogonal direction (Fig. 2.a).

Then, the iris region is unwrapped using the polar transform suggested by Daugman [2]. Its size is set to $256 \times 128$ pixels (Fig. 2.b).

Up to now, in our system, the occlusions have not been taken into account to generate the signature and have been processed as iris texture. So, the added segmentation step aims at detecting accurately the eyelid boundaries. 

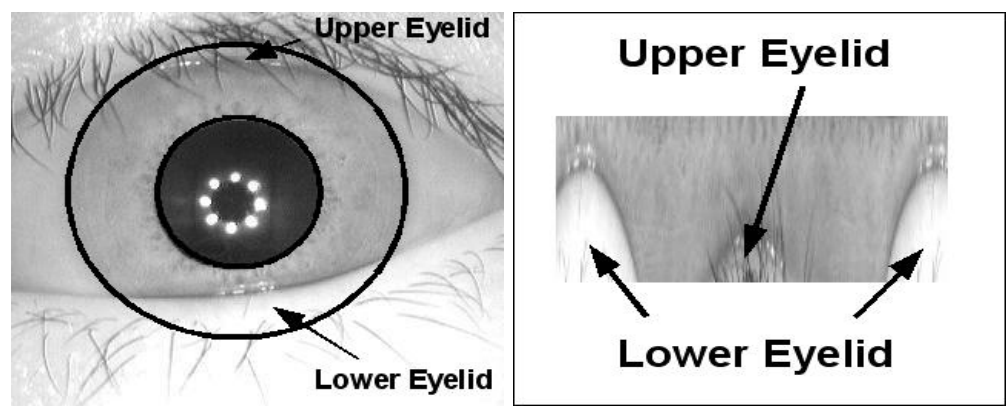

Fig. 2. a) Iris localization b) Unwrapped iris

\subsection{Eyelid Processing Method}

Most of the eyelid localization methods presented in the literature model eyelids as parabolic arcs. Daugman uses an integrodifferential operator with arcuate contour integration [2]. Wildes applies a Hough transform for the detection of parabolic arcs [4]. Chen uses a second-order curve fitting applied to the longest connected edge [5].

Eyelids, and especially the upper ones, are often crossed by eyelashes. Consequently, the eyelid boundary is often cut in several parts, and the longest detected edge does not always correspond to the eyelid boundary. Moreover, the edge detection step is generally very sensitive to illumination conditions, contrast, etc. Thus, it is impossible to tune the edge detector parameters in order to minimize bad connections, false detections or non detections in all cases.

That is why we propose a new method based on 4 steps: first, boundaries are enhanced by preprocessing. Then, a Canny edge detector [11] is applied on the preprocessed image, in order to get a map of edges. We use a priori information to suppress the ones that cannot correspond to eyelid boundaries. All the others are candidates to be eyelid edges, and are fitted with a parabolic curve. In the last step, we use a gradient maximisation in order to refine the parabola parameters and select the best one.

\subsection{Preprocessing}

We apply an anisotropic diffusion [10] in order to smooth the iris texture while keeping the eyelid boundaries.

Discretized anisotropic diffusion equation is presented as follow:

$$
I^{t+1}=I^{t}+\lambda\left[c_{N} \cdot \triangle_{N} I+c_{S} \cdot \triangle_{S} I+c_{E} \cdot \triangle_{E} I+c_{W} \cdot \triangle_{W} I\right] .
$$

where $0 \leq \lambda \leq 1 / 4$ for the numerical scheme to be stable, $\mathrm{t}$ indicates the iteration index, $\triangle$ indicates the nearest-neighbour differences for each direction $\mathrm{N}, \mathrm{S}, \mathrm{E}, \mathrm{W}$ and conduction coefficients $\mathrm{c}$ are function of this brightness gradient $\triangle$. We use $\lambda=0.25$ and 6 iterations. 

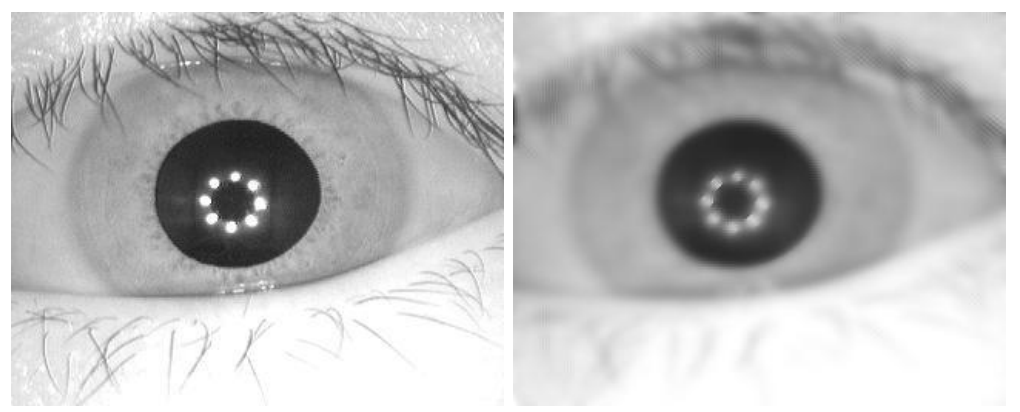

Fig. 3. a) Initial image b) Anisotropic diffusion preprocess

The results of the anisotropic diffusion (Fig. 3.b) are conform to our goal. Actually, the iris structures are smoothed while eyelid edges are almost not blurred.

\subsection{Edge Detection}

A Canny edge detection is performed on the preprocessed image (Fig. 4.a). The resulting image is binary, and provides a map of eyelid edge candidates.

In order to improve the speed and the robustness of the algorithm, we first restrict the analysis area to the inner iris. We also remove the left and the right parts of the iris in order to avoid connections between the eyelids/iris and the iris/sclera boundaries. The remaining edges above the pupil are candidates for the upper eyelid detection, while the ones below the pupil are candidates for the lower eyelid detection (Fig. 4.b).
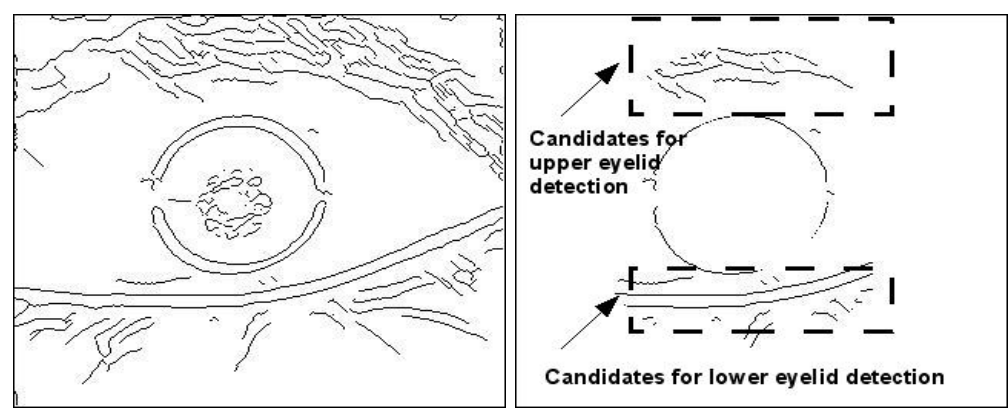

Fig. 4. a) Canny edge detection b) Restricted edge map

The mean length of all the remaining edges is then computed, and used as threshold to eliminate the smallest ones that come mainly from iris texture. 
Every candidate is fitted with a horizontally oriented parabolic curve, whose parametric equation is defined by two parameters, the vertex $S(X s, Y s)$ and the curvature $p$ :

$$
\begin{aligned}
& x=X s+2 p t^{2}, \quad t \in \Re \\
& y=Y s+2 p t
\end{aligned}
$$

A second selection stage is applied, introducing a priori knowledge about the form of the eyelids, i.e the direction of the parabola. The upper eyelids can be approximated by a parabolic curve whose curvature $p$ is positive. On the contrary, the curvature of the lower eyelid is negative. So, every fitted edge with an incorrect curvature $p$ is automatically eliminated (Fig. 5).

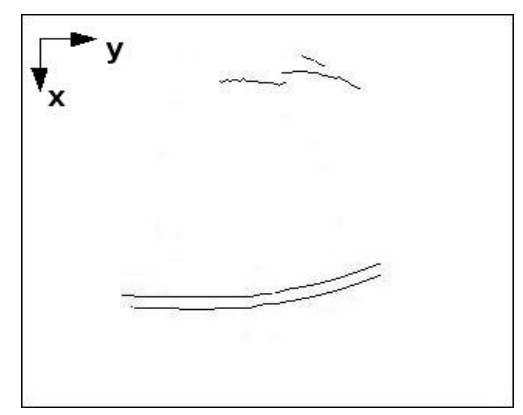

Fig. 5. Final edge candidates

At the end of this step, the edges that are likely to match the eyelid boundaries, have been extracted and modeled by a parabolic curve. Depending on the type of iris (little or highly textured), around 2 to 30 candidates still remain. We have also observed on many examples that the longest edge is not always the correct one.

The next step aims at refining the parameters of each curve and select the best one.

\subsection{Evaluation and Selection}

Let us denote by $p_{i}$ the curvature of the parabola equation for edge $i$ (Eq. 2). Each parabolic approximation is refined, by computing the gradient image along the curve for several curvatures.

A new gradient image $G$ is computed from the original image by applying a horizontal sobel operator in order to focus on the horizontal edges. Working on the original image is more suitable than working on the preprocessed one as the eyelid edges are not degraded. 
The gradient along the curve is then computed as follows:

$$
G^{(i)}\left(p_{i}\right)=\frac{1}{N_{i}} \sum G\left(x_{i}, y_{i}\right)
$$

In this equation, $N_{i}$ is the length of the edge $i . x_{i}$ and $y_{i}$ are the pixel coordinates along the parabolic curve for edge $i$ with curvature $p_{i}$. We limit the analysis in the horizontal direction to twice the iris size. The parameter $p_{i}$ is refined by calculating the gradient for several curvatures around $p_{i}$ and maximizing this gradient.

$$
G^{(i)}=\max _{j} G^{(i)}\left(p_{j}\right), p_{j} \in\left[0,2 p_{i}\right] .
$$

The final selected edge is the one maximizing this gradient, the method being applied for both upper and lower eyelids. An example is presented in (Fig. 6).

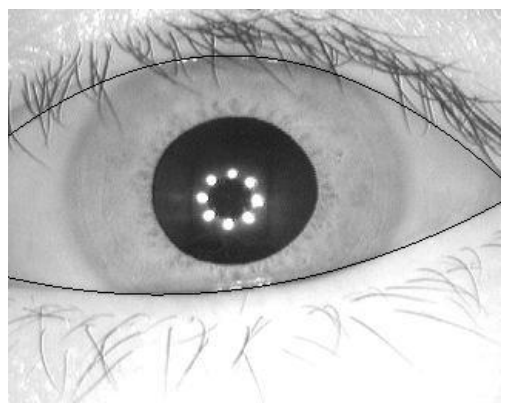

Fig. 6. Eyelids localization result

\section{Experiments and results}

We propose in this section an accurate and quantified performance evaluation of our eyelid localization method.

\subsection{Eyelid Testing Set}

The evaluation is performed on the CASIA-IrisV3-Interval database [12] which contain 396 classes and 2655 iris images. The entire database has been manually segmented as follows: operators have selected regularly spaced points along the occlusion boundaries. These points were then interpolated by a second-order curve. This segmentation is used as a reference to evaluate the reliability and the accuracy of the proposed segmentation algorithm. 


\subsection{Eyelids Localization Quality}

The evaluation is performed on the unwrapped images (section 3.1). Let us consider the binary unwrapped image, where the pixels equal to 1 belong to iris, and the pixels equal to 0 belong to the eyelid. It can be divided in two parts: the central part represents the upper eyelid localization, the side parts represent the lower eyelid localization. These two regions, denoted by $P 1$ and $P 2$ have the same size $S$ (16384 pixels).

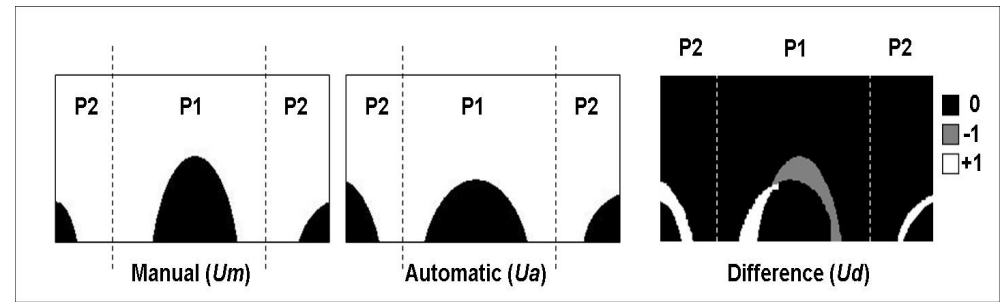

Fig. 7. Difference between manual and automatic segmentation

Using these conventions, we compute the difference between the manually $(U m)$ and the automatically segmented images $(U a)$. Let us denote by $U d$ the resulting image. The pixels equal to -1 are pixels classified as iris although they are in the eyelid region (sub-segmentation). Conversely, the pixels equal to +1 are pixels classified as eyelid, although they are in the iris region (over-segmentation). The sub-segmentation error $E_{s u b}^{p}$ and the over-segmentation error $E_{\text {over }}^{p}$ are defined as the ratio between the number of pixels which correspond respectively to -1 and +1 , and $S$ :

$$
\begin{aligned}
E_{\text {sub }}^{p_{i}} & =\left|\frac{\sum_{(x, y) \in p_{i}} U d(x, y)=-1}{S}\right| \\
E_{\text {over }}^{p_{i}} & =\left|\frac{\sum_{(x, y) \in p_{i}} U d(x, y)=+1}{S}\right|
\end{aligned}
$$

From equations (5) and (6), a global error $E^{p_{i}}$ is given:

$$
E^{p_{i}}=E_{\text {sub }}^{p_{i}}+E_{\text {over }}^{p_{i}}
$$

\subsection{Results}

Cumulative distribution functions are presented to estimate the performance of the upper and lower eyelid segmentation (Fig. 8). This graphic represent the proportion of images whose global error is lower than a given value. 

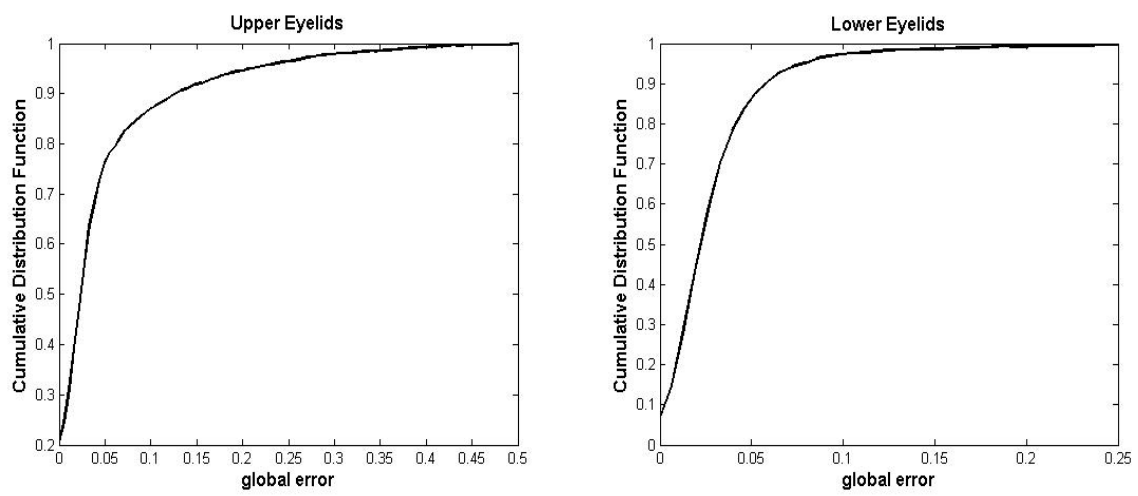

Fig. 8. Global Quality

The results (Fig. 8) show good performance for the lower eyelids. Indeed, we obtain $97.6 \%$ of localization with less than of $10 \%$ of global error. The performance of the upper eyelid localization is below. $87.5 \%$ of the upper localization have less than $10 \%$ of global error. Unlike lower eyelids which are well defined, upper eyelids suffer from important eyelashes presence. So, the main errors come from non detection or sub-segmentation.

However, the system is very robust to over-segmentation.

\section{Conclusion and Future Work}

In this paper, a new eyelid localization algorithm has been proposed. To this end, an edge detection method is performed on a restricted image area, leading to a set of possible boundary candidates. These edges are approximated by parabolic arcs. The parabola curvature of every candidate is refined using a gradient maximisation applied on a larger image area. Thus, the final boundaries can be selected and accurately modeled. A new performance measure, based on the comparison between manual and automatic segmentation has been proposed and provide accuracy and quality information. The obtained results show reliability of the proposed method.

This algorithm will be included in the preprocessing part, and will allow to restrict the signature comparison to the relevant coefficients. We will then study the link between the signature degradation (due to occlusions) and the recognition rates. From this, we will define an identification reliability indicator that will be used in a multimodal system, including face analysis [13].

\section{Acknowledgements}

We would like to thank the students who helped us to segment manually the eyelids. The manually segmented database is available upon request. 


\section{References}

1. Mansfield, T., Kelly, G., Chandler, D., Kane, J.: Biometric Product Testing Report. CESG/BWG Biometric Test Programme. National Physical Laboratory, UK (2001)

2. Daugman, J.: How Iris Recognition Works. IEEE Trans. CSVT,Vol. 14,no. 1,pp. 21-30, (2004)

3. Ma, L., Tan, T., Wang, Y., Zhang, D.: Personal Identification Based on Iris Texture Analysis. IEEE Trans. PAMI,Vol. 25,no. 12,pp. 1519-1533, (2003)

4. Wildes, R.P.: Iris recognition: An emerging biometric technology. Proceedings of the IEEE, Vol. 85,no. 9,pp. 1348-1363, (1997)

5. Chen, Y., Dass, S.C., Jain, A.K.: Localized Iris Image Quality Using 2-D Wavelet. Advances in Biometrics, International Conference, ICB 2006, Hong Kong, China, January 5-7, 2006, Proceedings. Lecture Notes in Computer Science 3832 Springer $2006373-381$

6. Cui, J., Wang, Y., Tan, T., Ma, L., Sun, Z.: A Fast and Robust Iris Localization Method Based on Texture Segmentation. Biometric Technology for Human Identification. Proceedings of the SPIE, Vol. 5404, pp. 401-408, 2004.

7. Sankowski, W., Grabowski, K., Napieralska, M., Zubert. M: Eyelids Localization Method Designed for Iris Recognition System. 14th International Conference on Mixed Design of Integrated Circuits and Systems, 2007.

8. Rydgren, E., Ea, T., Amiel, F., Rossant, F., Amara, A.: Iris Features Extraction Using Wavelet Packets. IEEE International Conference on Image Processing 2004, ICIP 2004, Singapore. Vol. 2,pp. 1348-1363, 2004

9. Rossant, F., Torres Eslava, M., Ea, T., Amiel, F., Amara, A.: Iris Identification and Robustness Evaluation of a Wavelet Packets Based Algorithm. IEEE International Conference on Image Processing 2005, ICIP 2005, Genova. Vol. 3,pp. 257-60, 2004

10. Perona, M., Malik, J.: Scale-Space and Edge Detection Using Anisotropic Diffusion. IEEE Transaction on Pattern Analysis and Machine Intelligence, Vol. 12,no. 7,pp. 629-639, July 1990

11. Canny, J.: A computational Approach to Edge Detection. IEEE Transaction on Pattern Analysis and Machine Intelligence, Vol. 8,no. 6,pp. 679-698, 1986

12. CASIA-IrisV3: http://www.cbsr.is.ac.cn/IrisDatabase.htm

13. Morizet, N., Amiel, F., Dris-Hamed, I., Ea, T.: A Comparative Implementation of PCA Face Recognition Algorithm. IEEE International Conference on Electronics, Circuits and Systems, December 11-14, 2007 - Marrakech, Morocco 